Jean-Claude Henquin Patrick Gilon

\section{ADRESSE}

J.C. Henquin : professeur à l'université de Louvain; P. Gilon : chargé de recherches du Fonds national de la recherche scientifique. Unité d'endocrinologie et métabolisme, UCL $55.30,55$, avenue Hippocrate, B-1200 Bruxelles, Belgique.

\title{
Le contrôle de la sécrétion d'insuline par le glucose: signaux déclenchants et amplificateurs
}

La sécrétion d'insuline par les cellules $\beta$ pancréatiques suit les variations des concentrations plasmatiques de glucose et d'autres nutriments. L'effet stimulant du glucose sur cette sécrétion dépend de son métabolisme à l'intérieur des cellules $\beta$ pancréatiques et est relayé par des signaux déclenchants et amplificateurs. L'élévation du rapport ATP/ADP cytoplasmique, provoquant la fermeture de canaux $\mathrm{K}^{+}$, entraîne la dépolarisation membranaire. Lorsque le potentiel de membrane atteint un certain seuil, les canaux calciques dépendants du voltage s'ouvrent, permettant une entrée intermittente de calcium. Les oscillations de la concentration cytoplasmique de $\mathrm{Ca}^{2+}$ qui en résultent déclenchent des oscillations synchrones de la sécrétion d'insuline. En outre, le glucose augmente l'effet du $\mathrm{Ca}^{2+}$ sur la sécrétion d'insuline, mais la nature de cette sensibilisation du système effecteur n'est pas élucidée.

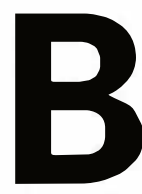

ien que les apports exogènes de substrats énergétiques soient intermittents, rythmés par les repas, la glycémie ne fluctue que dans des limites étroites chez l'homme normal. Cette stabilité est assurée par un système hormonal très efficace qui exerce des effets opposés sur certains organes qui stockent ou utilisent le glucose (et d'autres nutriments). Pendant les périodes de jeûne, plusieurs hormones dites contre-régulatrices (glucagon, catécholamines, glucocorticoïdes, hormone de croissance) stimulent la production endogène de glucose par la glycogénolyse et la néoglucogenèse afin de prévenir une hypoglycémie dangereuse pour le fonctionnement cérébral. En revanche, l'insuline est la seule hormone capable d'empêcher la glycémie de s'élever exagérément. En cas de carence en insuline ou de diminution de l'efficacité de l'hormone, les substrats exogènes ne sont plus stockés normalement et la production endogène de glucose (et d'autres substrats énergétiques directement utilisables) n'est plus freinée. Une hyperglycémie s'installe, caractéristique principale du diabète sucré.

\section{La cellule $\beta$ pancréatique,} un détecteur métabolique

L'insuline est synthétisée, stockée et sécrétée par les cellules $\beta$ pancréa- 


\section{RÉFÉRENCES}

1. Henquin JC. The cell biology of insulin secretion. In: Weir GC, Kahn CR, eds. The Joslin's diabetes mellitus. Philadelphia: Lea and Febiger, 1994: 56-80.

2. Portha B. Physiologie de la cellule $\boldsymbol{\beta}$ des îlots de Langerhans. médecine/sciences 1991 ; 7: 212-25.

3. Thorens B, Sarkar HK, Kaback HR, Lodish HF. Cloning and functional expression in bacteria of a novel glucose transporter present in liver, intestine, kidney, and betapancreatic islet cells. Cell 1988; 55 : 281-90.

4. Guerre-Millo M. Les transporteurs d'hexoses. médecine/sciences 1995; 11 : $1111-9$.

5. Matschinsky F, Liang Y, Kesavan P, Wang L, Froguel P, Velho G, Cohen D, Permutt MA, Tanizawa Y, Jetton TL, Niswender $K$, Magnuson MA. Glucokinase as pancreatic $\beta$-cell glucose sensor and diabetes gene. $I$ Clin Invest 1993 ; 92 : 2092-8.

6. Malaisse WJ. Regulation of insulin secretion by nutrients: the fuel concept. In: Flatt $\mathrm{PR}$, ed. Nutrient regulation of insulin secretion. London and Chapel Hill: Portland Press, 1992: 83-100.

7. Sekine N, Cirulli V, Regazzi R, Brown LJ, Gine E, Tamarit-Rodriguez J, Girotti M, Marie S, MacDonald MJ, Wollheim CB, Rutter GA. Low lactate dehydrogenase and high mitochondrial glycerol phosphate dehydrogenase in pancreatic $\beta$-cells. $J$ Biol Chem 1994; 269 : 4895-902.

8. Erecinska M, Bryla J, Michalik M, Meglasson MD, Nelson D. Energy metabolism in islets of Langerhans. Biochim Biophys Acta 1992; 1101 : 273-95.

9. Prentki M, Brun T, Roche E. Nutriments, insulinosécrétion et expression génique dans la cellule pancréatique $\beta$. Journées de Diabétologie de l'Hôtel-Dieu, 1994: 1-11.

10. Henquin JC, Debuyser A, Drews G, Plant TD. Regulation of $\mathrm{K}^{+}$permeability and membrane potential in insulin-secreting cells. In : Flatt PR, ed. Nutrient regulation of insulin secretion. London and Chapel Hill: Portland Press, 1992: 173-92.

11. Ashcroft FM, Rorsman P. Electrophysiology of the pancreatic $\beta$-cells. Prog Biophys tiques, qui sont localisées dans les îlots de Langerhans dispersés dans le parenchyme exocrine de la glande. La caractéristique principale de ces cellules $\beta$ pancréatiques est leur fonctionnement comme des «détecteurs métaboliques" [1]. Elles adaptent, en effet, la sécrétion d'insuline aux fluctuations de la glycémie et de la concentration plasmatique des autres nutriments (acides aminés, acides gras, corps cétoniques).

Dans la majorité des cellules, c'est la liaison d'un stimulus extracellulaire à un récepteur membranaire qui déclenche une réponse spécifique en modifiant la production de messagers intracellulaires. L'augmentation ou la diminution de la sécrétion d'insuline par des hormones ou des neurotransmetteurs ne font pas exception à la règle $[1,2]$. En revanche, le glucose et la plupart des autres nutriments ne se lient pas à un récepteur, mais exercent leurs effets en augmentant le métabolisme des cellules $\beta$. Par ces modifications de leur propre métabolisme, les cellules $\beta$ traduisent les variations de la concentration plasmatique des nutri- ments en signaux chimiques et biophysiques qui contrôlent la libération des granules d'insuline. Ce type de couplage stimulation-sécrétion est inhabituel mais sans doute pas spécifique des cellules $\beta$ pancréatiques. Il est vraisemblable que des mécanismes analogues interviennent dans le contrôle, par les nutriments, de la sécrétion des autres hormones du pancréas endocrine (glucagon, somatostatine) et de certaines hormones gastro-intestinales.

\section{Identification du glucose comme stimulus}

Le glucose entre dans les cellules $\beta$ par diffusion facilitée grâce à des transporteurs du type GLUT-2, semblables à ceux présents dans les hépatocytes $[3,4]$. Ce système de transport a une efficacité telle que les concentrations extracellulaire et cytoplasmique de glucose s'équilibrent très rapidement. Le glucose est alors phosphorylé, surtout par une glucokinase dont les propriétés permettent à la cellule d'adapter la vitesse de formation du glucose 6-phosphate aux

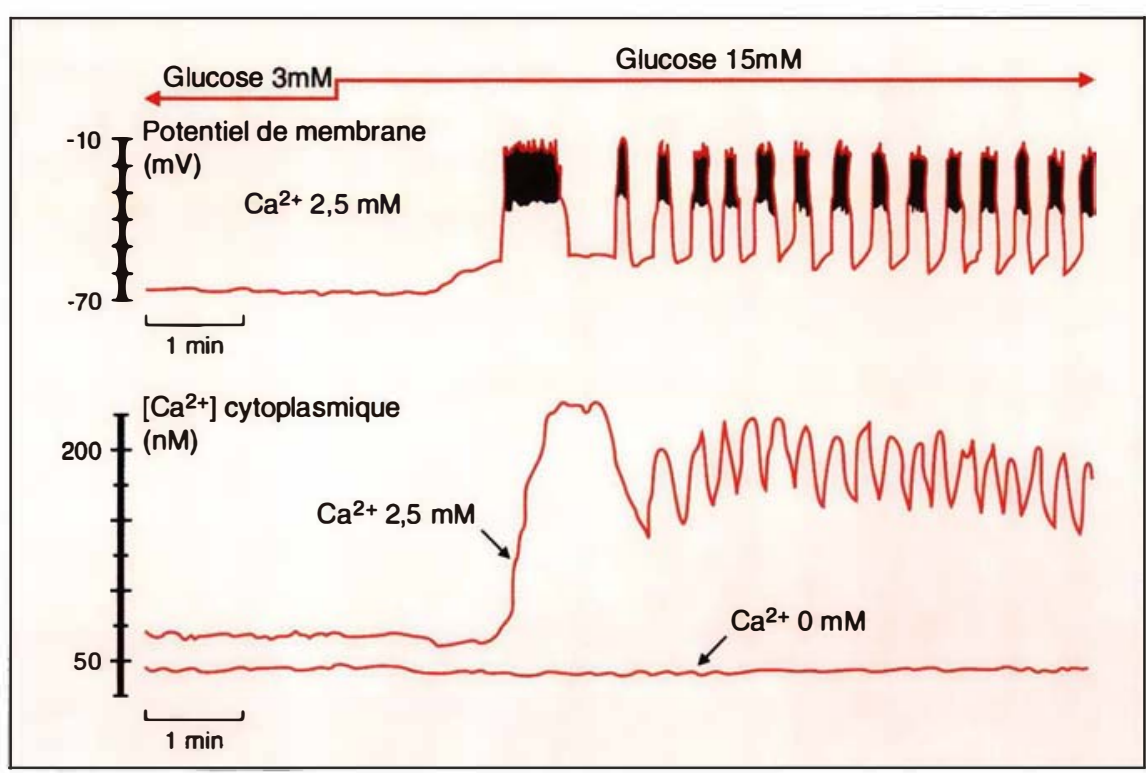

Figure 1. Influence du glucose sur le potentiel de membrane et la concentration cytoplasmique de $\mathrm{Ca}^{2+}$ des cellules $\beta$. Le potentiel de membrane d'une cellule $\beta$ au sein d'un îlot intact a été enregistré à l'aide d'une microélectrode intracellulaire. La concentration de $\mathrm{Ca}^{2+}$ cytoplasmique des cellules $\beta$ a été mesurée dans un îlot intact chargé avec du fura-2. La concentration de glucose du milieu a été augmentée de 3 à $15 \mathrm{mM}$ comme indiqué, alors que la concentration de $\mathrm{Ca}^{2+}$ restait constante $(2,5$ ou $0 \mathrm{mM})$. ( $D^{\prime}$ après [18].) 
variations de la concentration cytoplasmique de glucose et donc aux variations de la glycémie [5]. Bien que ces caractéristiques puissent faire considérer la glucokinase comme "le détecteur de glucose" des cellules $\beta$, l'existence d'autres sites de régulation du métabolisme ne doit pas être perdue de vue [6]. La destinée principale du glucose 6-phosphate est une conversion en pyruvate par la glycolyse qui s'accélère surtout par l'apport de substrats. Une particularité importante du métabolisme du glucose dans les cellules $\beta$ est la stimulation plus importante de la glycolyse oxydative que de la glycolyse totale. Cela peut être attribué à l'efficacité de la navette du glycérophosphate $[6,7]$ dont l'enzyme clé, la glycérophosphate déshydrogénase mitochondriale, est activée par le $\mathrm{Ca}^{2+}$. La réoxydation du NADH cytoplasmique par cette navette, plutôt que par la lactate déshydrogénase (avec conversion du pyruvate en lactate), permet l'oxydation d'une fraction importante du pyruvate dans le cycle de Krebs et une augmentation substantielle de la production d'ATP
[8]. Une autre partie du pyruvate est carboxylée et conduit à la formation de citrate puis de malonyl-CoA qui, en inhibant l'oxydation des acyl-CoA à longue chaîne, pourrait permettre à ceux-ci d'agir comme seconds messagers [9].

\section{Contrôle du potentiel de membrane}

A l'instar des cellules nerveuses ou musculaires, les cellules $\beta$ sont électriquement excitables. Toutefois, leur particularité est que ce sont les changements du métabolisme cellulaire qui contrôlent le potentiel membranaire. Par une technique de microélectrode intracellulaire, nous avons établi les caractéristiques de ce contrôle [10]. En l'absence de glucose, ou en présence d'une concentration non stimulante de glucose $(3 \mathrm{mM})$, le potentiel de membrane des cellules $\beta$ est stable, entre -60 et $-70 \mathrm{mV}$ (figure 1). Ce potentiel de repos est principalement déterminé par l'ouverture de canaux $\mathrm{K}^{+}$, dits sensibles à l'ATP (canaux $\mathrm{K}^{+}$-ATP), parce que l'application d'ATP à la face interne de la membrane plasmique (dans des expériences de patch-clam $p$ ) provoque leur fermeture $[10,11]$. Lorsque la concentration de glucose augmente, les canaux $\mathrm{K}^{+}$-ATP se ferment, la conductance pour le $\mathrm{K}^{+}$diminue et le potentiel de membrane (sous l'effet d'un courant non identifié parce que sans doute non spécifique) s'éloigne du potentiel d'équilibre du $\mathrm{K}^{+}$: la membrane se dépolarise. Lorsque le potentiel d'ouverture des canaux $\mathrm{Ca}^{2+}$ dépendants du voltage (de type L) est atteint, une activité électrique oscillatoire est déclenchée [10, 12-14]. Chaque oscillation est caractérisée par une dépolarisation rapide jusqu'à un potentiel de plateau au niveau duquel apparaissent des potentiels d'action, puis par une repolarisation rapide (figure 1). Ces oscillations du potentiel de membrane et les potentiels d'action correspondent à un influx de $\mathrm{Ca}^{2+}$ à travers les canaux $\mathrm{Ca}^{2+}$ dépendants du voltage. Lorsque la concentration de glucose augmente, ces phases d'activité électrique s'allongent pour aboutir à une dépolarisation permanente au-

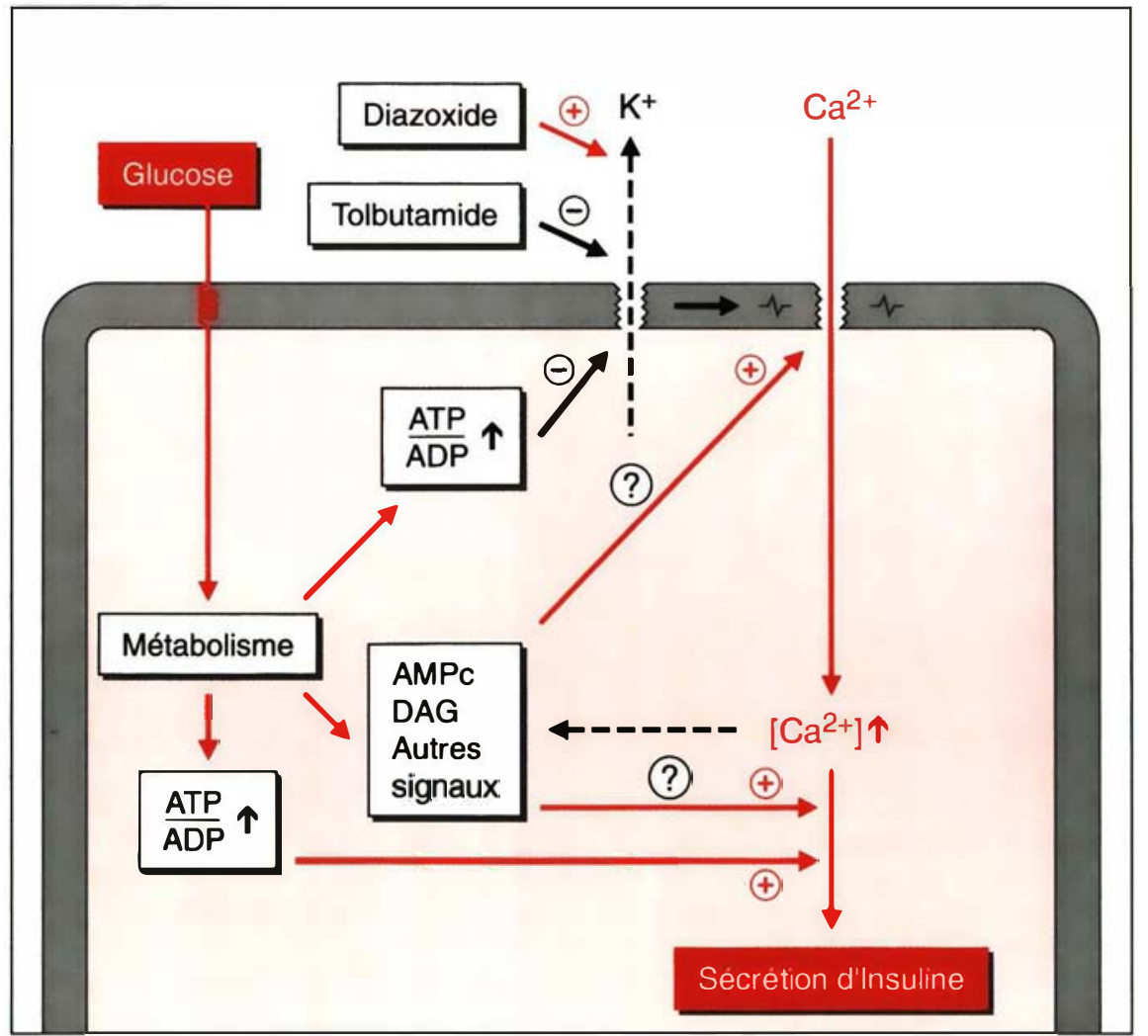

$m / s n^{\circ} 9$, vol. 11, seplembre 95
Figure 2. Représentation schématique des mécanismes de stimulation de la sécrétion d'insuline par le glucose. Les symboles $\oplus$ indiquent une stimulation, les symboles $\Theta$ une inhibition. Dès que la concentration de glucose augmente, le métabolisme des cellules $\beta$ s'accélère et l'augmentation du rapport ATP/ADP provoque la fermeture des canaux $K^{+}$sensibles à l'ATP. Cette fermeture entraîne une dépolarisation de la membrane, l'ouverture des canaux calciques dépendants du voltage (type $L$ ), un flux entrant intermittent de $\mathrm{Ca}^{2+}$, une élévation de la concentration cytoplasmique de $\mathrm{Ca}^{2+}$ $\left[\mathrm{Ca}^{2+}\right]_{i}$ et une stimulation de la sécrétion d'insuline. A côté de cette voie principale de production d'un signal déclenchant (l'élévation de la $\left.\left[\mathrm{Ca}^{2+}\right]_{i}\right)$, le métabolisme du glucose peut aussi, par l'intermédiaire de divers signaux, influer sur les canaux calciques et amplifier l'efficacité du $\mathrm{Ca}^{2+}$ surle système effecteur de la sécrétion. Le tolbutamide let les autres sulfamidés hypoglycémiants) stimulent la sécrétion d'insuline en fermant les canaux $\mathrm{K}^{+}$sensibles à l'ATP, alors que le diazoxide inhibe la sécrétion en ouvrant ces canaux. DAG : diacylglycérol. 


\section{RÉFÉRENCES}

12. Henquin JC. Les mécanismes cellulaires du contrôle de la sécrétion d'insuline. Arch Int Physiol Biochim 1990; 98: A61-A80.

13. Cook DL, Satin LS, Hopkins WF. Pancreatic $\beta$-cells are bursting, but how. Trends Neurosci 1991; 14: 411-4.

14. Nargeot J, Charnet P. Diversité moléculaire des canaux calciques: du gène à la fonction. médecine/sciences 1994; 10 : 1293308.

15. Pipeleers D, Kiekens R, Ling Z, Willikens A, Schuit F. Physiologic relevance of heterogeneity in the pancreatic beta-cell population. Diabetologia 1994; 37 (Suppl. 2): population

16. Detimary $P$, Henquin JC. Unmasking of large changes in adenine nucleotides in glucose-stimulated mouse islets. Diabetologia 1994; 37 (Suppl.1) : A1 1 (abstract).

17. Pavoine C, Pecker F. Lumière sur le calcium. médecine/sciences 1994; 10: 397-407.

18. Gilon P, Henquin JC. Influence of membrane potential changes on cytoplasmic $\mathrm{Ca}^{2+}$ concentration in an electrically excitable cell, the insulin-secreting pancreatic $\beta$ cell. J Biol Chem 1992; 267: 20713-20.

19. Santos RM, Rosario LM, Nadal A, Garcia-Sancho J, Soria B, Valdeolmillos M. Widespread synchronous $\left[\mathrm{Ca}^{2+}\right]$, oscillations due to bursting electrical activity in single pancreatic islets. Pflügers Arch 1991; 418 : 417-22.

20. Roe MW, Lancaster ME, Mertz. RJ, Worley JF III, Dukes ID. Voltage-dependent intracellular calcium release from mouse islets stimulated by glucose. J Biol Chem 1993 ; 268 : $9953-6$.

21. Gilon P, Shepherd RM, Henquin JC. Oscillations of secretion driven by oscillations of cytoplasmic $\mathrm{Ca}^{2+}$ as evidenced in single pancreatic islets. I Biol Chem 1993; $268: 22265-8$.

22. Hellman B, Gylfe E, Bergsten P, Grapengiesser E, Lund PE, Berts A, Tengholm A, Pipeleers DG, Ling Z. Glucose induces oscillatory $\mathrm{Ca}^{2+}$ signalling and insulin release in human pancreatic beta cells. Diabetolo- delà de $25 \mathrm{mM}$ glucose $[10,12]$. Les mécanismes responsables de ces changements sont complexes: une fermeture plus complète des canaux $\mathrm{K}^{+}$-ATP, une moindre inactivation des canaux $\mathrm{Ca}^{2+}$, ou une action du glucose sur d'autres canaux sont plausibles [10,13]. Par ailleurs, l'augmentation de l'activité électrique de chaque cellule $\beta$ sous l'effet de concentrations croissantes de glucose [10] montre clairement que le glucose provoque une gradation dans les réponses individuelles et pas seulement un recrutement de cellules actives [15].

Les changements de potentiel de membrane des cellules $\beta$ provoqués par le glucose dépendent étroitement de son métabolisme. L'hypothèse qui prévaut actuellement attribue la fermeture des canaux $\mathrm{K}^{+}$-ATP à une élévation du rapport des concentrations ATP/ADP dans le cytoplasme (figure 2). Nos mesures récentes de ce rapport en présence d'une large gamme de concentrations de glucose confortent cette hypothèse [16]. Néanmoins, toutes les objections [11] ne sont pas levées, en particulier parce que nous ignorons dans quelle mesure les phénomènes de consommation d'ATP (par les pompes ioniques, le cytosquelette...) peuvent influer sur la concentration locale d'adénine nucléotides à proximité des canaux. Il reste également possible que d'autres signaux issus du métabolisme influencent l'activité des canaux $\mathrm{K}^{+}-\mathrm{ATP}$, mais il ne s'agit ni de l'AMPc, ni du diacylglycérol dans des cellules $\beta$ normales. En revanche, l'activation des protéine kinases $\mathrm{A}$ et $\mathrm{C}$ pourrait influencer les canaux $\mathrm{Ca}^{2+}$ dépendants du voltage [10].

\section{Production d'un signal déclenchant}

Dans chaque îlot de Langerhans, les cellules $\beta$ (de 1000 à 5000 ) sont électriquement couplées par des jonctions communiquantes (gap junctions) et l'activité électrique que le glucose y déclenche est synchrone. Lors d'une augmentation rapide de la concentration ambiante de glucose, cette activité électrique présente un aspect biphasique, avec une première

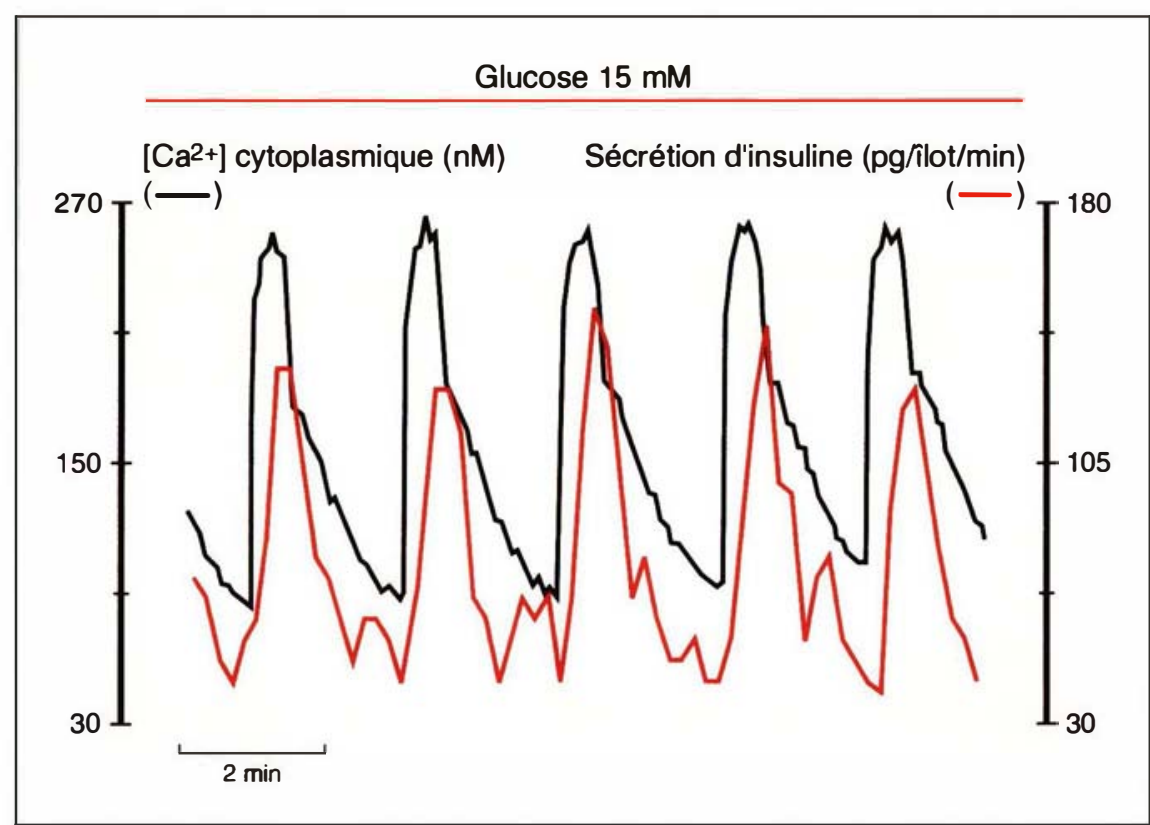

Figure 3. Corrélations entre les oscillations de la sécrétion d'insuline et les oscillations de la concentration cytoplasmique de $\mathrm{Ca}^{2+}$ dans les cellules $\boldsymbol{\beta}$. Les deux mesures ont été effectuées simultanément, à partir d'un même îlot de souris. La concentration de glucose $(15 \mathrm{mM})$ était maintenue constante. La concentration de $\mathrm{Ca}^{2+}$ dans le milieu était de $10 \mathrm{mM}$ lafin de ralentir le rythme des oscillations). (D'après [21].) 
phase plus ou moins longue et une seconde phase consistant en oscillations régulières et soutenues (/igure 1). Grâce à des indicateurs fluorescents [17], nous avons mesuré la concentration du $\mathrm{Ca}^{2+}$ ionisé, $\left[\mathrm{Ca}^{2+}\right]_{i}$, dans le cytoplasme des cellules $\beta$ au sein d'îlots de Langerhans intacts [18]. Les changements provoqués par le glucose sont semblables à ceux du potentiel de membrane: après une élévation initiale de longue durée, la $\left[\mathrm{Ca}^{2+}\right]_{\mathrm{i}}$ oscille de façon régulière aussi longtemps que la concentration de glucose reste élevée (figure 1). L'analyse des variations de la $\left[\mathrm{Ca}^{2+}\right]_{i}$ dans diverses zones de l'îlot montre que les oscillations sont synchrones mais que leur amplitude peut varier $[18,19]$. Il est donc bien établi que les oscillations du potentiel de membrane et de l'influx de $\mathrm{Ca}^{2+}$ entraînent des oscillations synchrones de la $\left[\mathrm{Ca}^{2+}\right]_{i}$ dans toutes les cellules $\beta$ de chaque îlot lors d'une stimulation par le glucose $[18,19]$, même si certains pools de $\mathrm{Ca}^{2+}$ intracellulaires pourraient aussi y contribuer [20]. En l'absence de calcium extracellulaire, une stimulation par le glucose entraîne une accélération du métabolisme cellulaire, une fermeture des canaux $\mathrm{K}^{+}$-ATP et une dépolarisation membranaire, mais pas d'influx de $\mathrm{Ca}^{2+}$, pas d'élévation de la $\left[\mathrm{Ca}^{2+}\right]_{\mathrm{i}}$ (le glucose ne mobilise pas le $\mathrm{Ca}^{2+}$ intracellulaire) (figure 1) et pas de sécrétion d'insuline. Il en est de même lorsque les canaux $\mathrm{Ca}^{2+}$ sont bloqués par des dihydropyridines ou d'autres antagonistes [18].

Récemment, nous avons réussi à mesurer simultanément les variations de la $\left[\mathrm{Ca}^{2+}\right]_{i}$ et de la sécrétion d'insuline dans un même îlot [21]. Des oscillations synchrones de la $\left[\mathrm{Ca}^{2+}\right]_{i}$ et de la sécrétion ont été mises en évidence lors d'une stimulation soutenue par $15 \mathrm{mM}$ glucose (figure 3). Ce synchronisme a également été observé lors de la première phase de la réponse au glucose et a persisté lors de changements spontanés ou induits de la fréquence des oscillations [21].

Que l'augmentation de la $\left[\mathrm{Ca}^{2+}\right]_{i}$ provoquée par une accélération de l'influx de $\mathrm{Ca}^{2+}$ constitue le signal déclenchant de la sécrétion d'insuline lors d'une stimulation par le glucose ne fait plus de doute. L'intérêt de la production d'un signal pulsatile est moins évident. Notre compréhension en est compliquée par le fait que les effets du glucose sur la $\left[\mathrm{Ca}^{2+}\right]_{i}$ diffèrent notablement dans des cellules $\beta$ tumorales ou même des cellules $\beta$ normales mais isolées [22] et dans la préparation plus physiologique d'îlots de Langerhans intacts. En outre, nous avons démontré la grande plasticité de ces oscillations de la $\left[\mathrm{Ca}^{2+}\right]_{i}[23]$. Le maintien d'îlots de souris normales en culture pendant un à quatre jours suffit à faire disparaître les oscillations de $\left[\mathrm{Ca}^{2+}\right]_{i}$ normalement induites par le glucose ou à en modifier profondément les caractéristiques (figure 4). Il sera important de déterminer quelles sont les répercussions de ces changements sur la sécrétion d'insuline.

\section{Les canaux $K^{+}-A T P$, une cible pharmacologique}

Le rôle essentiel que jouent les canaux $\mathrm{K}^{+}$-ATP dans le contrôle du potentiel de membrane des cellules $\beta$ et donc dans la production du signal déclenchant, explique que de nombreux agents pharmacologiques

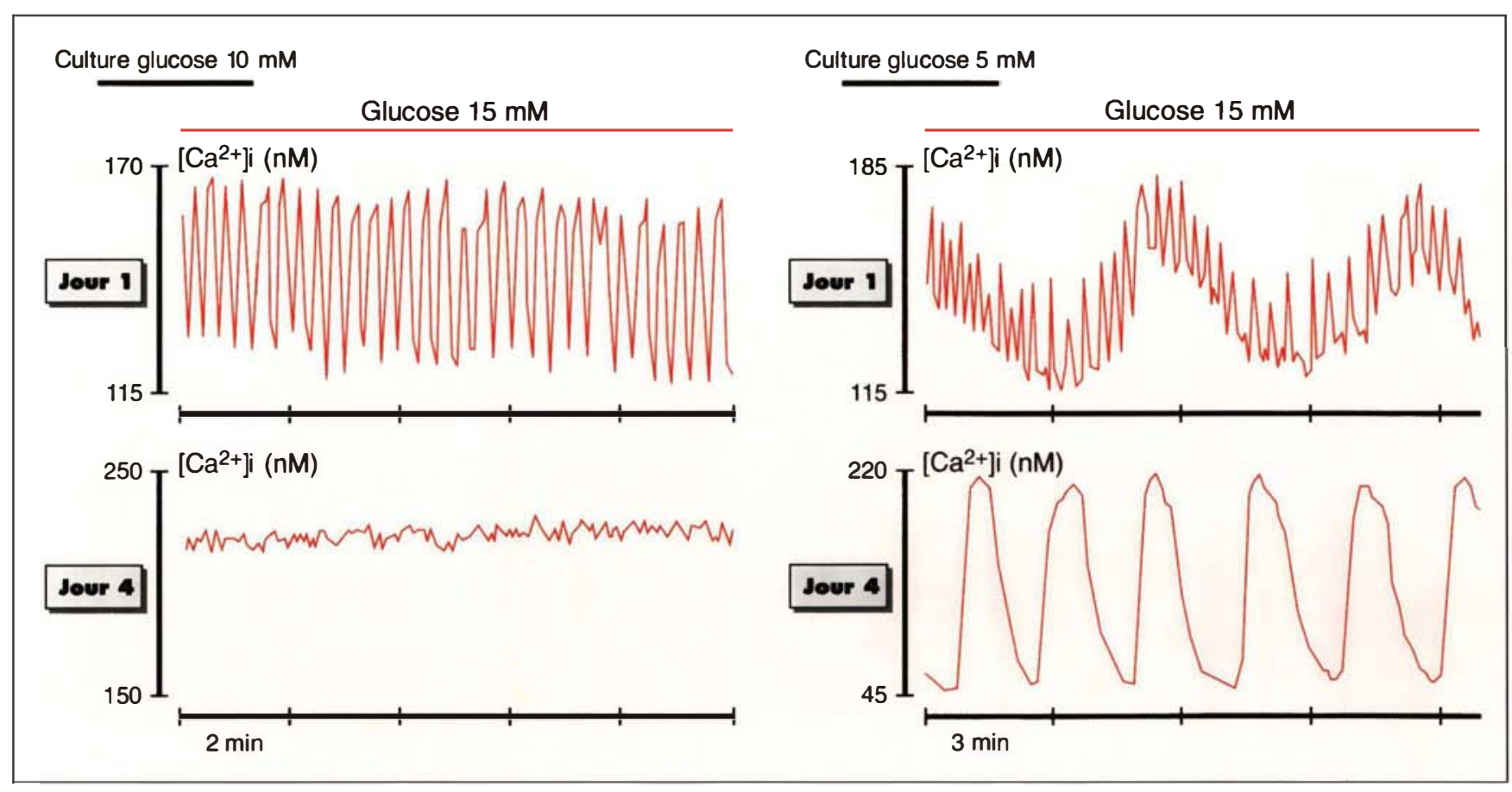

Figure 4. Plasticité des oscillations de la concentration cytoplasmique de $\mathrm{Ca}^{2+}$ dans les cellules $\boldsymbol{\beta}$. Des îlots de souris normales ont été maintenus en culture pendant un à quatre jours dans un milieu contenant 10 ou $5 \mathrm{mM}$ glucose. IIs ont ensuite été chargés avec du fura-2 pour mesurer les oscillations de la $\left[\mathrm{Ca}^{2+}\right]_{i}$ induites par $15 \mathrm{mM}$ glucose. (D'après [23].) 


\section{RÉFÉRENCES}

23. Gilon $P$, Jonas JC, Henquin JC. Culture duration and conditions affect the oscillations of cytoplasmic calcium concentration induced by glucose in mouse pancreatic islets. Diabetologia 1994 ; 37 : 1007-14.

24. Trube G, Rorsman P, Ohno Shosaku T. Opposite effects of tolbutamide and diazoxide on the ATP-dependent $\mathrm{K}^{+}$channel in mouse pancreatic beta-cells. Pflügers Arch 1986 ; 47: 493-9.

25. Ashford MLJ, Bond CT, Blair TA, Adelman JP. Cloning and functional expression of a rat heart $\mathrm{K}^{+}-\mathrm{ATP}$ channel. Nature 1994 . $370: 456-9$.

26. Aguilar-Bryan L, Nichols CG, Wechsler SW, Clement IVJP, Boyd IIIAE, Gonzalez G, Herrera-Sosa H, Nguy K, Bryan J, Nelson DA. Cloning of the $\beta$-cell high-affinity sulfonylurea receptor: a regulator of insulin secretion. Science 1995 ; 268: 423-6.

27. Henquin JC. Established, unsuspected and novel pharmacological insulin secretagogues. In: Bailey CJ, Flatt PR, eds. New antidiabetic drugs. London: Smith-Gordon, 1990: 93-106

28. Gembal M, Gilon P, Henquin JC. Evidence that glucose can control insulin release independently from its action on ATP-sensitive $\mathrm{K}^{+}$channels in mouse $\beta$-cells. J Clin Invest 1992; 89 : 1288-95.

29. Gembal M, Detimary P, Gilon P, Gao ZY, Henquin JC. Mechanisms by which glucose can control insulin release independently from its action on ATP-sensitive $\mathrm{K}^{+}$channels in mouse $\beta$-cells. J Clin Invest 1993; 91 : 87180 .

30. Sato Y, Aizawa T, Komatsu M, Okada N, Yamada T. Dual functional role of membrane depolarization $/ \mathrm{Ca}^{2+}$ influx in rat pancreatic $\beta$-cell. Diabetes 1992; $41: 438-43$.

31. Detimary $\mathbf{P}$, Gilon $\mathbf{P}$, Nenquin $\mathbf{M}$, Henquin JC. Two sites of glucose control of insulin release with distinct dependence on the energy state in pancreatic $\beta$-cells. Biochem J 1994; 297: 455-61.

32. Porte D. B-cells in type 2 diabetes mellitus. Diabetes $1991 ; 40: 166-80$.

33. O'Rahilly S, Turner RC, Matthews DR. Impaired pulsatile secretion of insulin in relatives of patients with non-insulin-dependent diabetes. N Engl J Med 1988; 318: 1225- affectent la sécrétion d'insuline en agissant à ce niveau.

Par une action directe, indépendante de changements du métabolisme cellulaire, le diazoxide ouvre les canaux $\mathrm{K}^{+}$-ATP [24]. Malgré la présence de glucose, cette ouverture provoque une repolarisation membranaire, un arrêt de l'influx de $\mathrm{Ca}^{2+}$, une diminution de la $\left[\mathrm{Ca}^{2+}\right]_{i}$ et une inhibition de la sécrétion d'insuline (figure 5). Le tolbutamide, au contraire, ferme les canaux $\mathrm{K}^{+}$-ATP par une action indépendante de changements du métabolisme. Cette propriété explique son action stimulante sur la sécrétion d'insuline et sa capacité de contrecarrer les effets inhibiteurs du diazoxide (figure 5).

Le tolbutamide est le prototype des sulfamidés hypoglycémiants utilisés pour augmenter la sécrétion d'insuline chez les diabétiques non insulinodépendants. Ils agissent tous par le même mécanisme: en se liant à un récepteur membranaire, ils provoquent la fermeture des canaux $\mathrm{K}^{+}$ATP (figure 2). Le clonage très récent de ces canaux [25] et du site de liaison des sulfamides hypoglycémiants

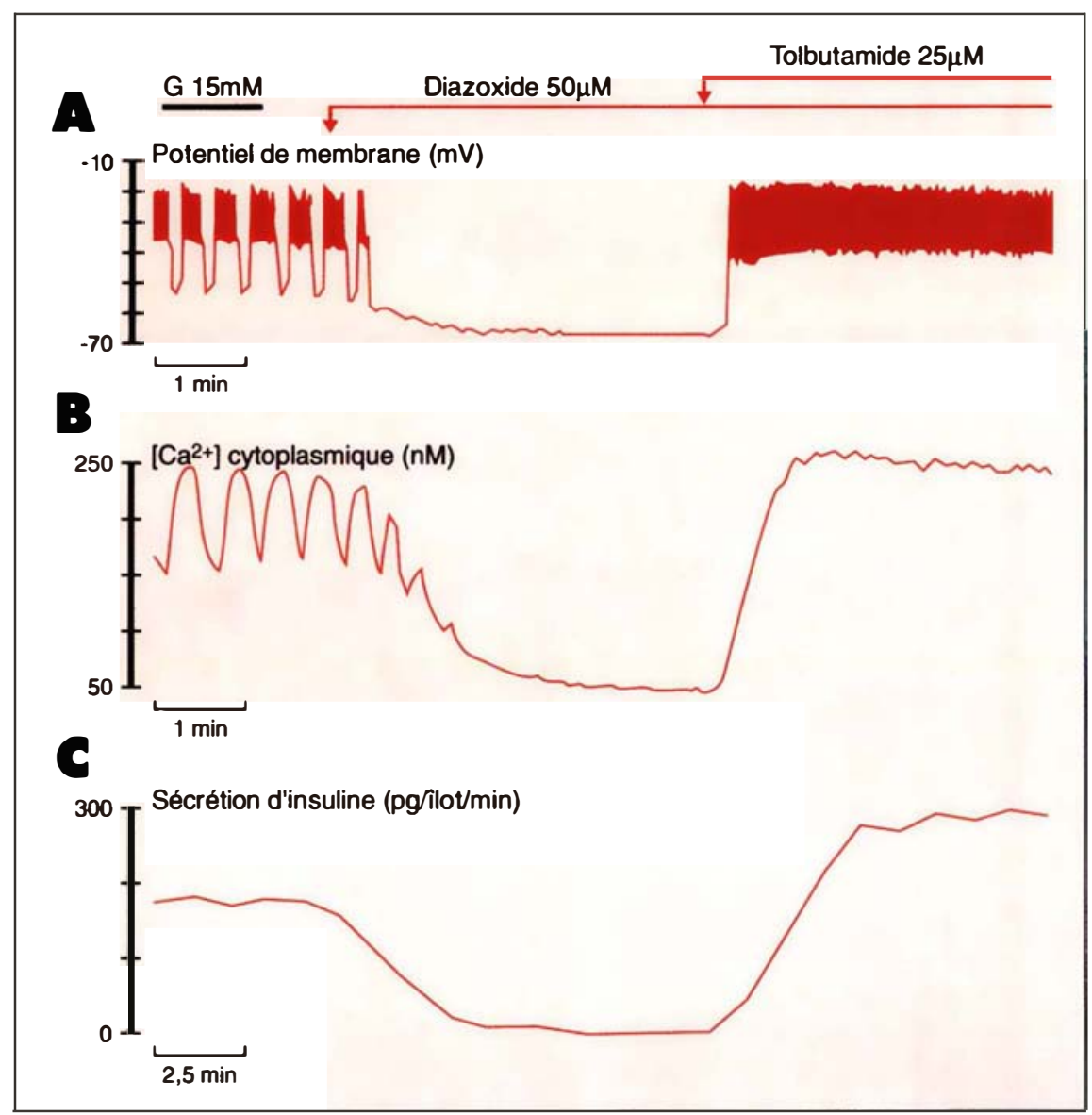

Figure 5. Importance des canaux $K^{+}$-ATP pour le contrôle de la sécrétion d'insuline par le glucose. Des îlots de souris ont été périfusés avec un milieu contenant $15 \mathrm{mM}$ de glucose (G). A. Le potentiel de membrane d'une cellule $\beta$ au sein d'un îlot intact a été enregistré à l'aide d'une microélectrode intracellulaire. B. La concentration de Ca ${ }^{2+}$ cytoplasmique $\left[\mathrm{Ca}^{2+}\right]_{i}$ des cellules $\beta$ a été mesurée dans un ilot intact chargé avec du fura-2. C. La sécrétion d'insuline a été mesurée à partir d'un groupe d'îlots. L'ouverture des canaux $\mathrm{K}^{+}-$ ATP par le diazoxide repolarise la membrane, abaisse la $\left[\mathrm{Ca}^{2+}\right]_{i}$ et inhibe la sécrétion d'insuline. La fermeture des canaux $K^{+}$-ATP par le tolbutamide renverse ces trois effets. (Adapté de [18].) 
[26] n'a pas encore permis d'établir avec certitude si le récepteur fait partie du canal lui-même ou est une protéine régulatrice associée. Par ailleurs, nos travaux ont établi que ces propriétés des sulfamidés hypoglycémiants sont partagées par de nombreuses substances pharmacologiques, de structure chimique parfois très différente [26]. De même, l'ouverture des canaux $\mathrm{K}^{+}$-ATP n'est pas l'apanage du diazoxide. Des agents, comme le pinacidil, actuellement développés comme antihypertenseurs, ont un effet semblable [26].

\section{Un signal amplificateur de la sécrétion}

Pendant longtemps les canaux $\mathrm{K}^{+}$ ATP de la membrane plasmique des cellules $\beta$ sont restés la seule cible identifiée sur laquelle le glucose pouvait agir pour contrôler la sécrétion d'insuline. Pour établir l'existence éventuelle d'autres cibles, il était donc nécessaire d'empêcher le glucose de fermer ces canaux. Cela est facilement réalisé avec le diazoxide, mais entraîne une inhibition complète de la sécrétion d'insuline (figure 5).

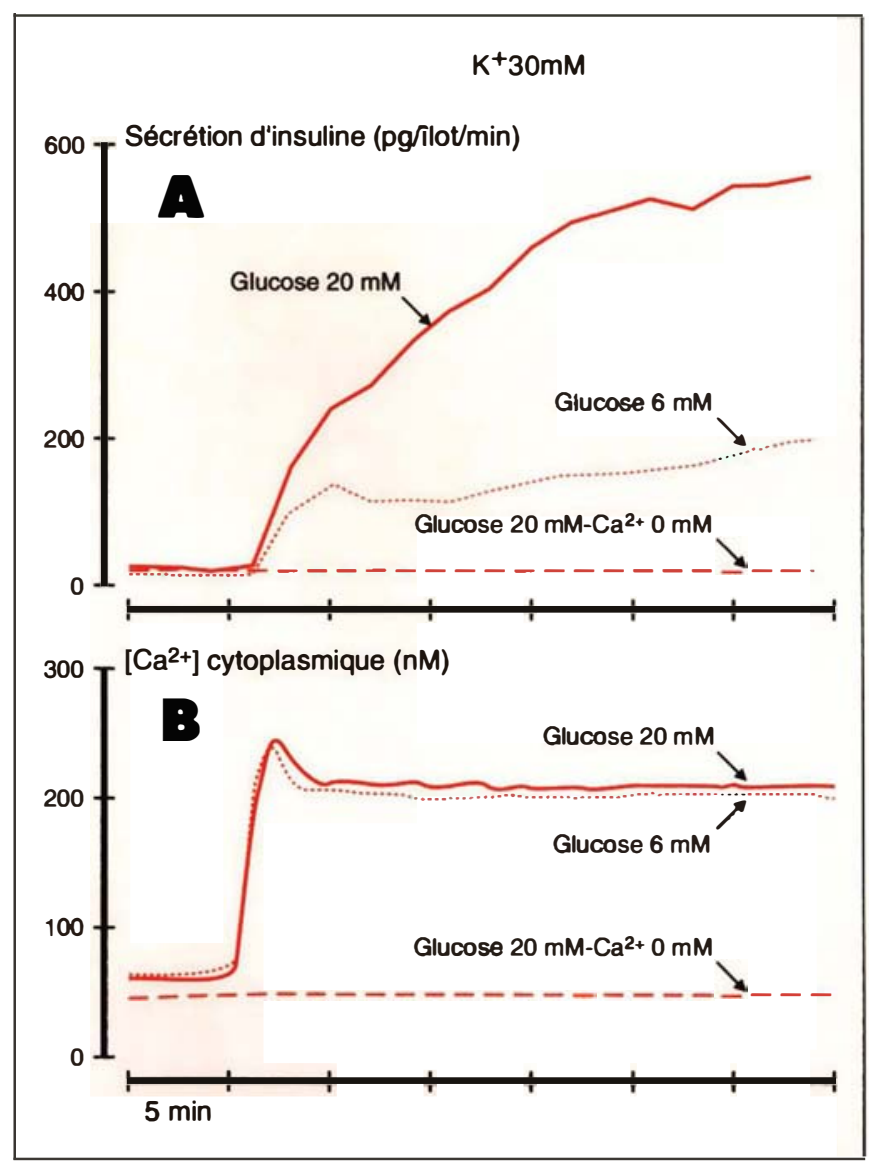

Figure 6. Production d'un signal amplificateur de la sécrétion d'insuline par le glucose (G). Des îlots de souris ont été périfusés avec un milieu contenant $250 \mu \mathrm{M}$ de diazoxide de façon à prévenir la fermeture des canaux $K^{+}$-ATP par le glucose. Le milieu contenait $20 \mathrm{mM}$ glucose et 2,5 $\mathrm{mM}$ de $\mathrm{Ca}^{2+}, 6 \mathrm{mM}$ de glucose et 2,5 $\mathrm{mM}$ de $\mathrm{Ca}^{2+}$ ou $20 \mathrm{mM}$ de glucose et pas de $\mathrm{Ca}^{2+}$. Pendant les cinq premières minutes, la concentration de $K^{+}$était de $4,8 \mathrm{mM}$ let la membrane des cellules $\beta$ était donc hyperpolarisée). La concentration de $K^{+} a$ ensuite été augmentée à $30 \mathrm{mM}$ (pour dépolariser la membrane). A. Changements de sécrétion d'insuline. B. Changements de la concentration cytoplasmique de $\mathrm{Ca}^{2+}$. Les deux paramètres ont été mesurés dans des expériences séparées. (Adapté de [29].)
Nous avons donc, en présence de diazoxide, dépolarisé la membrane en augmentant la concentration de $\mathrm{K}^{+}$ extracellulaire jusqu'à $30 \mathrm{mM}$, ce qui a pour effet de stimuler l'influx de $\mathrm{Ca}^{2+}$ et d'augmenter la $\left[\mathrm{Ca}^{2+}\right]_{\text {; }}$, donc de rétablir un signal déclenchant. Dans ces conditions, la sécrétion d'insuline reste contrôlée par les changements de la concentration de glucose [28-30].

Cette propriété du glucose est illustrée sur la figure 6. L'augmentation de la concentration de $\mathrm{K}^{+}$de 4,8 à $30 \mathrm{mM}$ stimule la sécrétion d'insuline trois fois plus en présence de $20 \mathrm{mM}$ que de $6 \mathrm{mM}$ glucose. Cette amplification requiert une élévation de la $\left[\mathrm{Ca}^{2+}\right]_{i}$ - elle ne survient pas en l'absence de $\mathrm{Ca}^{2+}$ extracellulaire -, mais ne résulte pas d'une élévation plus importante de cette concentration (figure 6). Nos observations démontrent que le glucose augmente l'efficacité du $\mathrm{Ca}^{2+}$ sur ses cibles intracellulaires. Cette propriété du glucose requiert son métabolisme par les cellules $\beta$ et est partagée par les autres nutriments métabolisés [28]. Le métabolisme du glucose conduit donc à la production d'un signal amplificateur de la sécrétion.

\section{Nature du signal amplificateur}

Il est bien connu que la sécrétion d'insuline peut être amplifiée par des mécanismes nerveux ou hormonaux $[1,2]$. C'est le cas au moment des repas, lorsque l'activation du système parasympathique libère de l'acétylcholine à proximité des cellules $\beta$ et lorsque l'arrivée des nutriments dans l'intestin libère des hormones comme le glucagon-like peptide 1 et le gastric-inhibitory polypeptide. Deux mécanismes principaux peuvent être mis en jeu dans cette amplification: une hydrolyse des phosphoinositides membranaires avec libération de $\mathrm{Ca}^{2+}$ intracellulaire par l'inositol-triphosphate et activation de la protéine kinase C par le diacylglycérol, ou une formation d'AMPc avec activation de la protéine kinase A $[1,2]$.

En revanche, l'amplification de la sécrétion par le glucose lui-même n'est pas due à une augmentation de l'AMPc, ni à une stimulation de l'hydrolyse des phospho-inositides et ne semble pas requérir l'activation de 
la protéine kinase C [29]. La nature du signal amplificateur grâce auquel le glucose augmente l'efficacité du $\mathrm{Ca}^{2+}$ sur la sécrétion n'est pas élucidée, en partie parce que notre connaissance des mécanismes par lesquels le signal déclenchant, le $\left[\mathrm{Ca}^{2+}\right]_{i}$, provoque l'exocytose des granules d'insuline est encore fragmentaire [1]. Plusieurs protéine kinases, notamment la protéine kinase dépendant du $\mathrm{Ca}^{2+}$ et de la calmoduline, le cytosquelette et sans doute de petites protéines $G$ interviennent. Une bonne corrélation existe entre l'effet amplificateur du glucose et le rapport ATP/ADP dans les îlots. Cette corrélation est étonuante, mais ne suffit pas à prouver qu'une relation causale existe entre les deux phénomènes. Un autre signal, issu du métabolisme et variant parallèlement au rapport ATP/ADP, pourrait constituer le véritable facteur de couplage (figure 2).

Néanmoins, l'intervention possible des adénine nucléotides, tant dans la production du signal déclenchant (vic les canaux $\mathrm{K}^{+}$-ATP) que dans l'amplification de la réponse sécrétoire, nous a conduits à comparer la sensibilité des deux étapes aux variations du rapport ATP/ADP. L'induction de variations de ce rapport par des poisons mitochondriaux a révélé que le contrôle du potentiel de membrane est beaucoup plus sensible à de petites variations de l'état énergétique des cellules $\beta$ que le mécanisme d'amplification [31]. Cela confirme la prééminence de la production du signal déclenchant dans le contrôle de la sécrétion d'insuline.

\section{Implications physiopathologiques et thérapeutiques}

Le diabète non insulinodépendant, la forme de loin la plus fréquente (90\%) du diabète sucré, résulte de la coexistence d'anomalies de la sécrétion d'insuline et de l'action de l'hormone sur ses tissus cibles. Parmi les anomalies les plus précocement détectables in vivo, on note: (1) une perte de la capacité du glucose d'induire une sécrétion rapide d'insuline, (2) une désorganisation des oscillations de la concentration plasmatique d'insuline et (3) une le glucose de la sécrétion d'insuline induite par des sécrétagogues non métabolisés [32, 33].

La première anomalie résulte sans doute d'un défaut dans la production du signal déclenchant par le glucose. En effet, le tolbutamide ou les autres sulfamidés hypoglycémiants peuvent corriger ce problème. Il pourrait en être de même de la deuxième anomalie, même s'il faut admettre que nous ignorons encore quel est le lien exact entre les oscillations relativement rapides de la sécrétion d'insuline au niveau de chaque îlot et les oscillations plus lentes de l'insulinémie. En revanche, la troisième anomalie est vraisemblablement la conséquence d'un trouble de la production du signal amplificateur. Plusieurs laboratoires ont entamé l'étude comparative de défauts possibles des signaux déclenchant et amplificateur dans les troubles de la sécrétion d'insuline par les cellules $\beta$ de modèles animaux du diabète non insulinodépendant. Si des défauts du signal amplificateur sont mis en évidence, notre attitude thérapeutique devra être revue. En effet, les sulfamidés hypoglycémiants, les seules armes actuellement utilisées pour augmenter la sécrétion d'insuline chez les diabétiques non insulinodépendants, peuvent corriger des anomalies de production du signal déclenchant, mais pas du signal amplificateur (figure 2).

\section{Conclusions}

Lors d'une stimulation par le glucose, le signal déclenchant la sécrétion d'insuline est une augmentation de la concentration de $\mathrm{Ca}^{2+}$ ionisé dans le cytoplasme des cellules $\beta$. Les variations de la concentration de glucose contrôlent la sécrétion en modulant l'amplitude et l'efficacité de ce signal. Par son action sur les canaux $\mathrm{K}^{+}-\mathrm{ATP}$, le glucose contrôle le potentiel membranaire, l'influx de $\mathrm{Ca}^{2+}$ et donc la concentration de $\mathrm{Ca}^{2+}$ dans le cytoplasme. Par ailleurs, le glucose sensibilise le système effecteur de la sécrétion à la stimulation par le $\mathrm{Ca}^{2+}$. Ces deux mécanismes requièrent le métabolisme du glucose et dépendent de l'état énergétique (rapport ATP/ADP) des cellules $\beta$. Hiérarchiquement, l'effet membranaire est le plus important parce qu'il permet la production d'un signal déclenchant, alors que le deuxième effet ne sert que d'amplificateur de la réponse sécrétoire

\section{Summary}

Triggering and amplifying signals in the control of insulin secretion by glucose

Pancreatic $\beta$ cells, which synthesize and secrete insulin, work as fuelsensors. They adapt the rate of insulin secretion to the variations in plasma levels of glucose and other nutrients. The stimulatory effect of glucosc requires metabolism of the sugar by $\beta$ cells to generate both triggering and amplifying signals. The closure of ATP-sensitive $\mathrm{K}^{+}$channels, probably mediated by an elevation in the ATP/ADP ratio in the cytoplasm, leads to membrane depolarization. The subsequent opening of voltage-dependent $\mathrm{Ca}^{2+}$ channels during bursts of action potentials produces an intermittent $\mathrm{Ca}^{2+}$ influx that causes an oscillatory rise of cytoplasmic $\mathrm{Ca}^{2+}$. This triggering signal entrains synchronous oscillations of insulin secretion. In addition, glucose increases the effectiveness of $\mathrm{Ca}^{2+}$ on the secretory machinery. The nature of this amplifying signal is still unclear, but adenine nucleotides might also be involved. Hierarchically, the control of the membrane potential by glucose metabolism is the most important site of regulation, because it modulates the production of the triggering signal, whereas the second site only serves to amplify the response.

\section{Remerciements}

Les travaux réalisés dans le laboratoire des auteurs ont bénéficié du soutien financier du Fonds de la recherche scientifique médicale. du Fonds national de la recherche scientifique et du ministère de la Politique scientifique, à Bruxelles.

\section{TIRÉS À PART}

J.C. Henquin. 